\title{
Formación de profesores de matemáticas en contextos de diversidad
}

\author{
Mathematic Teachers Training in contexts of Diversity
}

Luis Alexander Castro Miguez ${ }^{1}$

\section{Resumen}

Se ha reflexionado sobre la importancia de la formación de profesores de matemáticas en los procesos educativos de los estudiantes en contextos de diversidad, de tal manera que se favorezca y superen la visión de instituciones educativas que, marginan del sistema educativo a poblaciones vulnerables. Esta reflexión se realizó como estudiante del Doctorado Interinstitucional en Educación de la Universidad Distrital "Francisco José de Caldas" en la revisión de antecedentes correspondiente al proyecto de investigación que fue de carácter exploratorio con un enfoque mixto y multimetódico. Los procesos de formación son un componente esencial en el desarrollo profesional de los profesores, de ahí el énfasis en las disciplinas de la individualidad y de un trabajo fuera de la escuela a un desarrollo profesional colectivo y desde las instituciones educativas, en el cual se reconozca a cada uno de los estudiantes como sujetos activos en su proceso de formación y transformación proactivos y por ende proponer opciones educativas distintas que posibiliten excelencia de aprendizaje y donde la constante sea eliminar las barreras de todo tipo que pueden limitar su presencia, aprendizaje, participación y promoción en la vida escolar, con particular atención a aquellos más vulnerables, aspecto que responde en parte a las necesidades que se plantean desde una educación inclusiva.

Palabras clave: Diversidad; educación; formación; profesor; matemáticas.

\section{Summary}

The importance of training mathematics teachers in the students' educational processes in diversity contexts has been reflected on, in such a way that the vision of educational institutions that marginalize vulnerable populations from the educational system is favored and exceeded. This reflection was made as a student of the Interinstitutional Doctorate in Education of "Francisco José de Caldas" University District in the background review corresponding to the research project that was exploratory in nature with a mixed and multi-method approach. Training processes are an essential component in the professional development of teachers, hence the emphasis on the disciplines of individuality and work outside of school to collective professional

\footnotetext{
1 Magister en docencia de las matemáticas. Estudiante del Doctorado Interinstitucional en Educación de la Universidad Distrital Francisco José de Caldas, Bogotá (Colombia). Email: lacastrom@correo.udistrital.edu.co - ORCID: https://orcid.org/oooo-0003-0993-4988

Recibido: 21/06/2019 Aprobado: 19/03/2019
}

Castro Miguez, L. (2020). Formación de profesores de matemáticas en contextos de diversidad. Ciencia E Interculturalidad, 26(01), 36-49. https://doi.org/10.5377/rci.v26io1.9882 
development and from educational institutions, in which it is recognized each of the students as active subjects in their proactive training and transformation process and therefore propose different educational options that enable learning excellence and where the constant is to eliminate barriers of all kinds that limit their presence, learning, participation and promotion in school life, with particular attention to those most vulnerable, an aspect that responds in part to the needs that arise from an inclusive education.

Keywords: Diversity; Education; Training; Professor; Mathematics.

\section{Introducción}

Los profesores están llamados a repensar respecto a sus prácticas pedagógicas, donde el reconocimiento del otro (estudiante, docente, directivo, padre de familia y comunidad educativa) desde su diferencia, cobra una vital importancia en todo el sistema educativo. Se hace necesario una transformación de las relaciones con el aprendiz y por ende proponer opciones educativas distintas que permitan reconocer sus condiciones de aprendizaje, identificando sus diferentes formas de comunicar, sentir, percibir, planificar, interactuar, etc.

El artículo presenta algunas tensiones, necesidades y campos de problemas de investigación desde la formación de profesores de matemáticas en un ámbito nacional e internacional. La investigación es de carácter exploratorio con un enfoque mixto y multimetódico, pretende aportar a la discusión actual en relación con los componentes curriculares pertinentes en el proceso de formación de los profesores que acogen en sus aulas diversidad de poblaciones. Su intervención, desde su rol como profesional de la educación, debe procurar que se teja una estructura escolar en donde todos los estudiantes "reciban todo el apoyo necesario considerando sus diferencias en los ritmos de aprendizaje, intereses, expectativas y necesidades específicas" (Á. López, Calderón, Escalante, Sáiz, y León, 2016, p. 40); si todo ello es permeado por un componente afectivo y se procuran ambientes de aprendizaje accesibles, quizás podría aportarse a una educación matemática más incluyente.

\section{Metodología}

Reconocer como prioridad la formación continuada de profesores de matemáticas y no sólo las sugerencias de expertos en cursos de formación, muchas veces lejanas de la problemática concreta del aula escolar al atender poblaciones en situación de vulnerabilidad; fenómeno que implica todo un sistema dinámico en el que están presentes diferentes tipos de relaciones, protagonistas y procesos asociados. Por la naturaleza compleja del fenómeno y de acuerdo con lo planteado por Hernández, Fernández y Baptista (2014) este proyecto de investigación se sitúa en un enfoque mixto que, emplea para su estudio procesos sistemáticos, empíricos y críticos desde 
la recolección y análisis de datos cuantitativos y cualitativos; además, se reconoce con Cazau (2006) que, de acuerdo a su alcance, es de carácter exploratorio. Su estructura, trata de explorar desde diferentes perspectivas teóricas, en el diseño e implementación de una estrategia de formación de profesores de matemáticas, los efectos que tiene la incorporación de procesos reflexivos sobre el reconocer, el repensar y el reconstruir las prácticas de enseñanza de las matemáticas en ambientes de aprendizaje accesibles como escenarios naturales (Wieringa, 2014).

\section{Desarrollo}

\section{Formación de profesores de matemáticas}

La formación de profesores de matemáticas en Colombia es una preocupación en las últimas décadas impregnada por diferentes corrientes teóricas provenientes de las Escuelas Normales Superiores, las Facultades de Educación y el paradigma de la matemática moderna; además de la Ley General de Educación, los lineamientos curriculares, los estándares básicos de competencias, los derechos básicos de aprendizaje y más recientemente las mallas de aprendizaje formuladas para la básica primaria. León et al. (2014) afirma que, algunas de estas "propuestas aún continúan sin ser incorporadas en la generalidad de las instituciones educativas de básica y media y en las instituciones formadoras de docentes" (p. 63).

Específicamente los programas de formación de profesores de matemáticas en su mayoría, toman como referencia en la organización de sus currículos algunos en los componentes: disciplina, pedagógico, didáctico, tecnológico, investigativo, práctica, contexto profesional y diversidad; "con menor peso sobre la formación son, en su orden, el tecnológico con 4.94\%, y el más crítico, la diversidad con 1.01\%" (León et al., 2014, p. 64), lo que hace evidente que éstos pueden ser posibles campos de reflexión en la formación de profesores de matemáticas en Colombia.

Guacaneme, Obando, Garzón y Villa-Ochoa (2013), destacan que a pesar de que existen estudios sobre la formación de profesores, se hace necesario consolidar colectivos de trabajo en los que su objeto de estudio sea la formación de profesores de matemáticas. El proyecto ALTER-NATIVA reconoce que los "Referentes curriculares con incorporación de tecnologías para la formación del profesorado de matemáticas en y para la diversidad" fruto de por lo menos cinco aspectos fundamentales: "las múltiples experiencias con lo matemático y su didáctica; las múltiples representaciones de lo matemático y de su didáctica; los múltiples tipos de interacción en los ambientes de aprendizaje; las poblaciones en vulnerabilidad educativa por sus condiciones sensoriales, étnicas o económicas y una metodología de interacción y desarrollo definida por las comunidades de práctica" (León et al., 2014, p. 11), se ofrecen como respuesta a las necesidades identificadas cuando se procura el desarrollo de una educación matemática con y para todos. 
Por último, León et al. (2017), desde los resultados en el proyecto "Desarrollo didáctico y tecnológico en escenarios didácticos para la formación de profesores que acogen la diversidad" al que se incorporó la perspectiva teórica del diseño universal para el aprendizaje y una posición ético-política de una educación matemática con todos, afirman que es necesario continuar con investigaciones que permitan fortalecer la formación de profesores de matemáticas para el acogimiento de la diversidad de poblaciones presentes en el aula escolar.

En una mirada internacional es posible afirmar que, en las últimas dos décadas algunas investigaciones sobre formación de profesores, tanto inicial como continuada, permiten identificar diferentes fortalezas y necesidades. Gómez (2005) afirma que, la formación de profesores de matemáticas puede tener un núcleo común para conceptualizar el conocimiento del profesor de matemáticas y fundamentar los programas de formación. Llinares (2007), reconoce la necesidad de definir la formación de profesores de matemáticas a partir de la práctica de enseñar matemáticas, referentes que permiten crear oportunidades para generar procesos de desarrollo profesional.

Alsina, Planas y Calabuig (2009), reconocen en el modelo de formación activa -el aprendizaje reflexivo, un aporte relevante a la formación de profesores de matemáticas puesto que a través de éste es posible la reconstrucción y construcción de las competencias profesionales del profesor de matemáticas, permitiéndole comprender la complejidad en general y de las matemáticas en particular. Godino y Batanero (2009) afirman que, en la formación didáctica del profesor de matemáticas "la reflexión sobre la propia experiencia matemática experimentada es necesaria para la apropiación y adaptación de los conocimientos didácticos por parte del profesor" (p. 1). Pochulu, Font y Rodríguez (2016), reconocen una vez más que la formación del profesor de matemáticas constituye un campo de investigación relevante cuyo interés, en la última década, se ha centrado en el conocimiento y las competencias que necesitan los profesores para enriquecer los procesos educativos de las matemáticas.

Por lo anterior puede afirmarse que, la formación de profesores de matemáticas es objeto de investigación en el campo de la educación matemática (L. Blanco y Contreras, 2012; Sáenz y Lebrija, 2014; León et al., 2014; Gil, 2015; Pochulu et al., 2016; León y Suárez, 2016; León et al., 2017), por su influencia tanto en las prácticas escolares como en la práctica profesional de los profesores de matemáticas; además, se reconoce como prioridad dar continuidad al proceso de formación desde las propias "necesidades y opiniones de los mismos profesores y no sólo de las sugerencias de expertos en cursos de formación genéricos, muchas veces ajenos a la problemática concreta del aula del profesor en formación" (Sáenz y Lebrija, 2014, p. 237). Se destacan como campos de problemas de investigación desde la formación de profesores, los siguientes: 


\section{La diversidad de poblaciones en las aulas, componente curricular relevante}

Al no reconocer las condiciones de aprendizaje de algunos estudiantes (indígenas, sordos, negros, mestizos, niños de la calle, ciegos, emigrantes, etc.), éstos podrían encontrarse en situación de vulnerabilidad con el riesgo de ser marginados de todo proceso educativo. Los profesores quizás no cuentan con las herramientas pertinentes que permitan dar respuesta a las necesidades emergentes ante esta diversidad social, racial, étnica, religiosa, de género y de habilidades; reconocida esta problemática algunos investigadores se plantean preguntas tales como: ¿qué se debe hacer para desarrollar escuelas que puedan efectivamente "tender la mano" a todos los niños y jóvenes, sean cuales sean sus circunstancias y características personales?, ¿qué proponen las investigaciones sobre la forma en que los docentes y las escuelas podrían desarrollar prácticas más inclusivas?, (Ainscow et al., 2013; Spratt y Florian, 2015).

Definitivamente todos los sujetos tienen la oportunidad de ser reconocidos desde y en un grupo sociocultural por sus diferencias y potencialidades; es aquí donde la sociedad en general y el sistema educativo en particular debe aunar esfuerzos que permita garantizar a toda la población un espacio de calidad que favorezca los procesos educativos, intentando dar respuesta a preguntas como: ¿Cuál ha de ser el rol del Estado y de la sociedad para garantizar el derecho a una educación de calidad sin exclusiones?, ¿qué factores contribuyen al desarrollo de sistemas educativos y escuelas más inclusivas? (R. Blanco, 2008; Ainscow, 2011) tienen diferentes experiencias de educación y abandonan las escuelas con resultados muy diferentes. En muchos países, la mayoría de los niños más pobres son los que más pierden, alcanzan los peores resultados y asisten a escuelas de rendimiento más bajo.

Es necesario que tanto facultades de educación superior como instituciones educativas estén abiertas a la atención de la diversidad, donde la reflexión de las prácticas de enseñanza sea uno de los mecanismos que les permita afrontar este desafío y quizás replantear algunos de los procedimientos que se han venido implementando (Echeita y Ainscow, 2011; León et al., 2014; Ainscow, 2016; Florian y Pantić, 2017).

\section{La diversidad en el aula y las nuevas competencias matemáticas}

Si las diferencias de cualquier tipo se excluyen del aula de clase, se pierde la valiosa oportunidad de aprendizaje que se tiene al reconocer la diversidad de ideas, gustos, intereses, habilidades y necesidades de aprendizaje de todos los estudiantes. Estos aspectos hacen que emerjan algunas preguntas: ¿Cómo pueden progresar en su aprendizaje estudiantes "diferentes" a la mayoría de los que asisten a una escuela (diferentes por varios motivos, porque tienen alguna discapacidad, porque son de culturas distintas y no dominan la lengua predominante, porque pertenecen a un entorno social marginado) en un aula en la cual cada uno trabaja sólo en su pupitre y en la cual el profesor o la profesora debe atender individualmente a sus estudiantes 
tan "diversos" unos de otros?, ¿cómo pueden progresar estos alumnos "diferentes" en un aula en la cual los estudiantes compiten entre ellos para lograr ser el primero, el mejor, sea como sea? (Pujolàs, 2009; Kefallinou, 2016).

Diferentes estudios reconocen que existen contenidos formativos en la formación de profesores que se centran en el análisis de las distintas situaciones presentes en los procesos educativos que pueden surgir como consecuencia de la diversidad presente en el aula; sin embargo, aún es necesario continuar indagando sobre este aspecto, en particular sobre la formación continuada de profesores desde alguna de las siguientes preguntas: ¿cómo garantizar un aprendizaje a lo largo de la vida profesional con una orientación inclusiva?, ¿qué conocimiento necesitan los maestros para responder más eficazmente a la diversidad en sus aulas? (López y Aké, 2015; Pantić y Florian, 2015; Florian y Pantić, 2017).

La atención a la diversidad presente en el aula de clase debe constituirse en el eje transversal de la formación de profesores, convirtiendo a las instituciones educativas en entidades promotoras de un cambio en donde no exista fronteras entre la inclusión y la exclusión de poblaciones; pues todos tienen la posibilidad de vivenciar un aprendizaje de calidad (Hart, Drummond y McIntyre, 2007; R. Blanco, 2008; Florian, 2015).

\section{Evaluación del impacto de formación de profesores}

Pueden limitar el poder conocer los logros de los estudiantes en el desarrollo de sus competencias básicas; sin embargo, también la eficiencia de las instituciones educativas, la coordinación interinstitucional, el funcionamiento de las escuelas, el trabajo de los docentes, entre otros aspectos; como lo afirma Sáenz y Lebrija (2014; p. 221), "la formación no se ha acompañado de un proceso de investigación que, desde un marco teórico específico, evalúe el impacto de los programas formativos en las prácticas de aula de los profesores", hace falta diseñar nuevos modelos y estrategias de acción y nuevas formas de cooperación que favorezcan los procesos de formación de profesores; esto implica preguntarse por ¿cómo organizar la formación de profesores en torno al desarrollo de procesos reflexivos que surgen deductiva o inductivamente de la teoría y de aquéllos que nacen abductivamente, a partir de la reflexión sobre la práctica?, ¿cómo motivar a los docentes para llevar a cabo un cambio de paradigma que transite del conocimiento teórico tradicional sobre las metodologías de enseñanza, al desarrollo de competencias que se alimentan de la práctica?, ¿dónde se sitúan sobre el continuo de una profesionalidad técnica-reflexiva-crítica, y hasta qué punto se estaría propiciando un modelo de docente consumidor o creador de conocimiento? (Hevia, 2013; Escudero, González, y Rodríguez, 2018). 


\section{La modernidad en contraste con probable obsolescencia del currículum}

Obliga a los profesores a reajustar constantemente el currículum, a enriquecer sus prácticas de enseñanza, a generar ambientes de aprendizaje que impliquen el uso pedagógico de las Tecnologías de la Información y la Comunicación, procurando así entornos más dinámicos, accesibles e interactivos, etc. Esto hace necesario la existencia de procesos reflexivos por parte de los profesores sobre su propia experiencia y sobre los procesos educativos experimentados para la apropiación y adaptación de los conocimientos didácticos; este "análisis y reflexión didáctica requiere dominar y aplicar herramientas conceptuales y metodológicas adecuadas" (Godino y Batanero, 2009, p. 1). Es por esto que una estrategia de formación de profesores de matemáticas, basada en el aprendizaje reflexivo puede favorecer un cambio hacia una enseñanza de las matemáticas "centrada en el aprendiz" (Alsina et al., 2009; Sáenz y Lebrija, 2014; Ametller y Alsina, 2017).

Ahora, si desde la formación de profesores de matemáticas se procura un reconocimiento de las capacidades y potencialidades que tiene la población estudiantil presente en el aula de clase, quizás se podrá realizar una gestión curricular pertinente con la población implicada y dar respuesta a preguntas como: ¿Cuáles pueden ser las formas de una formación eficaz si ésta se juzga como útil y si se tiene en cuenta que la ambición no es formar especialistas en didáctica, sino formar profesores capaces de utilizar de manera pertinente los aportes de la didáctica?, ¿de qué manera las Tecnologías de la Información y la Comunicación contribuyen en el proceso educativo de poblaciones diversas?, ¿cómo integrar las experiencias de alumnos que en principio pueden ser muy diferentes de las experiencias a las que el profesor está acostumbrado? (Adler, Ball, Krainer, Lin y Novotna, 2005).

\section{La poca articulación entre teoría y práctica de las matemáticas}

Hace que "el docente no llegue a establecer un vínculo entre lo que percibe en la realidad del aula y lo que proviene del saber teórico" (Alsina y Palacios, 2010, p. 1); los procesos de formación de profesores de matemáticas debe reconocer las prácticas de enseñanza como fuentes de constante aprendizaje y es por esto que "la persona que se forma lo hace dando significado a unos contenidos y no recibiendo esos mismos ya impregnados de significado" (Alsina y Palacios, 2010, p. 2). Hasta aquí es prudente preguntarse por aspectos como ¿Cuáles son las variables o dimensiones que caracterizan los contextos y los problemas que se abordan en la formación de profesores de matemáticas?, ¿cómo es y cómo puede ser la formación de los profesores para enseñar matemáticas en la educación básica y media? (Flores, 2004; Saravia y Flores, 2005; Gómez-Chacón y Planchart, 2005; Gil, 2015).

Aportar a la articulación entre la teoría y la práctica de enseñar matemáticas desde procesos reflexivos sobre el diseño de actividades, implica tener presente las 
condiciones de aprendizaje de cada uno de los estudiantes y que las mismas hacen parte de un sistema que reconoce sus niveles de complejidad, de ahí que sea posible formular preguntas como: ¿Sobre qué tópicos pueden discutir los profesores cuando están planificando cada uno de los temas?, ¿es posible encontrar organizadores para este nivel de reflexión sobre el currículo de matemáticas, además de los contenidos? (Gómez, 2005; Guacaneme et al., 2013; Pochulu et al., 2016).

\section{Diferentes capacidades, intereses, ritmos, motivaciones y experiencias de aprendizaje de las matemáticas}

Invita a replantear muchas de las acciones presentes en un aula de clase que intenta homogenizar sus procesos educativos, y hacer explícito, desde la formación continuada de profesores, diferentes herramientas didácticas que permitan afrontar este desafío. Planas (2003) afirma que, todo estudiante "debería recibir una educación matemática significativa y ninguno debería ser excluido por motivo de diferencias culturales, sociales o lingüísticas" (p. 24), pero ¿qué ayudas y recursos son necesarios para facilitar el aprendizaje de todos los alumnos?, ¿cómo movilizar el conocimiento de un sujeto?, ¿en qué tipo de prácticas queremos que nuestros alumnos participen? (Planas, 2003; Planas, 2005; Bishop, Tan y Barkatsas, 2015).

Una de estas herramientas puede partir desde lo planteado por Civil, Planas, y Fonseca (2000), en cuanto al desarrollo de comunidades de aprendices en el aula donde el aprendizaje tiene lugar mediante la participación de todos en las actividades propuestas a través de su interacción, responsabilizando al profesor de planificar cuidadosamente el diseño de cada una de las actividades educativas; previendo las posibles ayudas que los estudiantes necesitarán durante el proceso de aprendizaje (Gómez y Lupiáñez, 2007; Clements y Sarama, 2015).

Planas (2003) afirma que, "la diversidad de culturas ya es un hecho. Pero ¿qué ocurre con la cultura de la diversidad?” (p. 24). Es importante afrontar dos grandes retos desde la educación matemática: conseguir un "aprendizaje matemático para todos y cuestionar aquellas formas de enseñanza que no contribuyen a la democratización de un aprendizaje de calidad" (Planas, 2003, p. 35). Además, de identificar los factores educativos que reducen las oportunidades de aprendizaje matemático de algunos estudiantes y superar dichas barreras desde el diseño de ambientes de aprendizaje accesibles (León et al., 2014; León, Alfonso, Bravo, Romero, y López, 2018). Si las estrategias de formación de profesores procuran, siguiendo a Hopkins y Stern (1996, citados por Durán y Giné, 2011): compromiso, afecto, conocimiento de la didáctica de la materia enseñada, múltiples modelos de enseñanza, reflexión sobre la práctica y trabajo en equipo que promueva el aprendizaje entre los colegas; es posible "liderar y fundamentar procesos de formación e implementación de políticas educativas para la formación de profesores para atender contextos de diversidad" (León et al., 2014, p. 13). 


\section{Conclusiones}

Pensar en una educación accesible e inclusiva implica reconocer que, una de las misiones de la escuela es la de proporcionar el apoyo necesario para alcanzar una vida de calidad en la que todo el mundo tiene la misma oportunidad de aprender; para ello debe implementarse estrategias que permitan romper las barreras que están impidiendo, obstaculizando, cerrando la oportunidad de que todas las personas puedan acceder con equidad y justicia al sistema educativo y social. Estas estrategias deben procurar ambientes de aprendizaje accesibles, infraestructuras adecuadas, cambios fundamentales en la política educativa, garantizar una formación de profesores, reconocer las diferentes herramientas tecnológicas a emplear en el proceso educativo, entre otros aspectos, que permita garantizar condiciones de asequibilidad, accesibilidad, aceptabilidad y adaptabilidad.

La formación de profesores jugará un papel importante al reconocer las condiciones de aprendizaje de estudiantes en contextos de diversidad, favoreciendo el desarrollo de una educación con y para todos y superando así, la visión de instituciones educativas que marginan poblaciones vulnerables del sistema educativo; de ahí que sea necesario pensar en planes de estudio y estrategias que, promuevan una educación para todos, respetando a los estudiantes en sus singularidades y el desarrollo de capacidades que les ayuden a transformar la realidad en la que viven, tanto en el entorno escolar, como en la familia y la comunidad. Son entonces las necesidades de los estudiantes que deben orientar las políticas públicas de inclusión (Melo y Geller, 2015). A medida que la educación inclusiva se incorpora cada vez más a los discursos de política internacional, puede ser imperativo explorar e identificar teorías e ideas que puedan responder a contextos diversos y muy desiguales de escolarización (Danforth y Naraian, 2015).

Por todo lo anterior se reconoce que la formación de profesores es un componente esencial en el desarrollo profesional de estos, de ahí que deba pasar del énfasis en las disciplinas, de la individualidad y de un trabajo fuera de la escuela a un desarrollo profesional colectivo y desde las instituciones educativas. (G. García, 2016) se reconoce que, una de las herramientas imprescindibles para aportar a un sistema educativo que trabaja desde las diferencias, la inclusión y la accesibilidad es la formación continuada de profesores, ya sea en estrategias pedagógicas o reflexión sobre sus prácticas de enseñanza en contextos de diversidad.

\section{Agradecimientos}

El autor agradece al Doctorado Interinstitucional en Educación, al Grupo de Investigación GIIPL y M., a la Comisión Europea y a los socios del proyecto: ERASMUS+: Higher Education - International Capacity Building - ACACIA - (561754-EPP-1-20151-CO-EPKA2-CBHE-JP), http://acacia.digital. 
Esta publicación obtuvo el financiamiento de: El Fondo de Asistencia Internacional de los Estudiantes y Académicos Noruegos (SAIH).

\section{Lista de referencia}

Adler, J., Ball, D., Krainer, K., Lin, F.-L. y Novotna, J. (2005). Reflections on an emerging field: Researching mathematics teacher education. Educational Studies in Mathematics, 60, 359-381. https://doi.org/10.1007/s10649-005-5072-6

Ainscow, M. (2011). Responding to the challenge of equity within education systems. Ediciones Universidad de Salamanca, 17, 73-87.

Ainscow, M. (2016). Diversity and Equity: A Global Education Challenge. New Zealand Journal of Educational Studies, 51, 143-155. https://doi.org/10.1007/ s40841-016-0056-x

Ainscow, M., Dyson, A., Goldrick, S. y West, M. (2013). Promoting equity in education. Revista de Investigación en Educación 11(3), 32-43.

Alsina, À. y Palacios, C. (2010). ¿Cómo mejorar la educación matemática del profesorado en activo? Aula, (196), 61-66.

Alsina, À., Planas, N. y Calabuig, T. (2009). El aprendizaje reflexivo en la formación del profesorado de matemáticas. En M. Tortosa, J. Álvarez y N. Pellín (Eds.), VII Jornadas de Redes de Investigación en Docencia Universitaria: la calidad del proceso de ensenanza/aprendizaje universitario desde la perspectiva del cambio (pp. 252-257). Alicante: Universidad de Alicante.

Ametller, J. y Alsina, À. (2017). ¿Qué aportan el aprendizaje reflexivo y la enseñanza dialógica a la formación permanente? Un primer análisis con profesorado de ciencias y de matemáticas. Enseñanza de las ciencias: Revista de investigación y experiencias didácticas, 2059-2064.

Bishop, A., Tan, H. y Barkatsas, T. (Eds.). (2015). Diversity in mathematics education. Towards inclusive practices. New York: Springer. https://doi.org/10.1080/1479 4802.2016.1141114

Blanco, L. y Contreras, L. (2012). Conceptualizando y ejemplificando el conocimiento matemático para la enseñanza. Revista Iberoamericana de Educación Matemática, 30, 101-123. 


\section{EDUCACIÓN}

Blanco, R. (2008). Marco conceptual sobre educación inclusiva. En Conferencia Internacional de Educación. La educación inclusiva: El camino hacia el futuro (pp. 5-14). Ginebra: UNESCO.

Cazau, P. (2006). Introducción a la investigación en ciencias sociales (3.a ed.). Buenos Aires: Universidad de Buenos Aires.

Civil, M., Planas, N. y Fonseca, D. (2000). La atención a la diversidad en el aula de matemáticas: hacia una participación pedagógica y matemática. Revista Uno, 23, 29-43.

Clements, D. y Sarama, J. (2015). El Aprendizaje y la Enseñanza de las Matemáticas a Temprana Edad. Traducido por León, O., Lange, A, León, L. y Toquica, A.

Danforth, S. y Naraian, S. (2015). This new field of inclusive education: Beginning a dialogue on conceptual foundations. Intellectual and Developmental Disabilities, 53(1), 70-85. https://doi.org/10.1352/1934-9556-53.1.70

Durán, D. y Giné, C. (2011). La formación del profesorado para la educación inclusiva: Un proceso de desarrollo profesional y de mejora de los centros para atender la diversidad. Revista Latinoamericana de Educación Inclusiva, 5(2), 153-170.

Echeita, G. y Ainscow, M. (2011). La educación inclusiva como derecho. Marco de referencia y pautas de acción para el desarrollo de una revolución pendiente. Tejuelo, 12, 26-46.

Escudero, J., González, M. y Rodríguez, M. (2018). Los contenidos de la formación continuada del profesorado: ¿qué docentes se están formando? Educación Siglo XXI, 21(1), 157-180.

European Agency for Special Needs and Inclusive Education. (2016). Raising the achievement of all learners in inclusive education - Literature Review. (A. Kefallinou, Ed.). Odense, Denmark.

Flores, I. (Ed.). (2004). ¿Cómo estamos formando a los maestros en América Latina? En Encuentro internacional. El desarrollo profesional de los docentes en América Latina. Lima: Programa de Educación Básica de la Cooperación Alemana al Desarrollo.

Florian, L. (2015). Conceptualising Inclusive Pedagogy: The Inclusive Pedagogical Approach in Action. En J. Deppeler, T. Loreman, R. Smith y L. Florian (Eds.), Inclusive Pedagogy Across the Curriculum, (International Perspectives on Inclusive 
Education, Volumen 7) (pp. 211-234). Melbourne: Emerald. https://doi. org/10.1108/S1479-363620157

Florian, L. y Pantić, N. (Eds.). (2017). Teacher education for the changing demographics of Schooling. Issues for research and pracice. Cham: Springer.

García, G. (2016). El camino hacia la educación inclusiva de las niñas y niños con discapacidad en el Derecho Internacional de los Derechos Humanos (Tesis Doctoral). Universidad Carlos III de Madrid.

Gil, D. (2015). La formación de los docentes de matemáticas en Colombia. En B. García (Ed.), Escuela y educación superior: temas para la reflexión (pp. 151-170). Bogotá: Universidad Distrital Francisco José de Caldas.

Godino, J. y Batanero, C. (2009). Formación de profesores de matemáticas basada en la reflexión guiada sobre la práctica. En VI Congreso Iberoamericano de Educación Matemática-CIBEM (pp. 4-9). Puerto Montt.

Gómez-Chacón, I. y Planchart, E. (Eds.). (2005). Educación Matemática y Formación de Profesores. Propuestas para Europa y Latinoamérica. Bilbao: Universidad de Deusto.

Gómez, P. (2005). Diversidad en la formación de profesores de Matemáticas: En la búsqueda de un núcleo común. Revista EMA, 10(1), 1-40.

Gómez, P. y Lupiáñez, J. (2007). Trayectorias hipotéticas de aprendizaje en la formación inicial de profesores de matemáticas de secundaria. PNA, 1(2), 79-98.

Guacaneme, E., Obando, G., Garzón, D. y Villa-Ochoa, J. (2013). Informe sobre la Formación inicial y continua de Profesores de Matemáticas: El caso de Colombia. Cuadernos de Investigación y Formación en Educación Matemática, 11-49.

Hart, S., Drummond, M. y McIntyre, D. (2007). Learning without Limits: constructing a pedagogy free from determinist beliefs about ability. En L. Florian (Ed.), The Sage handbook of special education (pp. 449-514). London: Sage. https://doi. org/10.2304/forum.2004.46.3.5

Hernández, R., Fernández, C. y Baptista, P. (2014). Metodología de la investigación (6.a ed.). México: Mc Graw Hill Education.

Hevia, R. (Ed.). (2013). Formación continua y desarrollo profesional docente. En Ponencias del seminario internacional (p. 170). Santiago: Organización de Estados Iberoamericanos para la Educación, la Cienca y la Cultura - OEI. 


\section{EDUCACIÓN}

León, O., Alfonso, G., Bravo, F., Romero, J. y López, H. (2018). Ambientes de Aprendizaje. (F. Bravo y O. León, Eds.). Bogotá: Proyecto ACACIA.

León, O., Bonilla, M., Romero, J., Gil, D., Correal, M., Ávila, C., ... Márquez, H. (2014). Referentes curriculares con incorporación de tecnologías para la formación del profesorado de matemáticas en y para la diversidad. (Á. López y M. Borja, Eds.) (1.a ed.). Bogotá: Universidad Distrital Francisco José de Caldas.

León, O., Romero, J., Carranza, E., Sánchez, F., Suárez, W., Castro, C. y Gil, D. (2017). Arquitectura de validación de diseños didácticos para la formación de profesores de matemáticas. Revista Colombiana de Educación, 73, 235-26o. https://doi.org /10.17227/01203916.73rce233.258

León, O. y Suárez, W. (2016). Cartografía de programas de formación de profesores de matemáticas en colombia. Revista Horizontes Pedagógicos, 18(2), 120-132.

Llinares, S. (2007). Formación de profesores de matemáticas. Desarrollando entornos de aprendizaje para relacionar la formación inicial y el desarrollo profesional. En Conferencia invitada en la XIII Jornadas de Aprendizaje y Enseñanza de las Matemáticas - JAEM (pp. 1-12). Granada.

López, Á., Calderón, D., Escalante, I., Sáiz, M. y León, O. (2016). Modelo de validación ALTER-NATIVA de objetos virtuales de aprendizaje en escenarios naturales. (O. León y Á. López, Eds.). Bogotá: Universidad Distrital Francisco José de Caldas.

López, J. y Aké, L. (2015). Formación matemática del docente de Educación Especial. Educación Matemática en las Américas: 2015. Necesidades Especiales., 14, 94-101.

Melo, G. y Geller, M. (2015). Ensino de Ciências e Políticas Públicas de Educação Inclusiva: um estudo teórico. En X Encontro Nacional de Pesquisa em Educação em Ciências - X ENPEC (pp. 1-8).

Pantić, N. y Florian, L. (2015). Developing teachers as agents of inclusion and social justice. Education Inquiry, 6(3), 333-351. https://doi.org/10.3402/edui.v6.27311

Planas, N. (2003). Medidas de apoyo pedagógico, didáctico y organizativo ante el fenómeno del fracaso matemático escolar en alumnos minoritarios. SUMA, 42, 23-36.

Planas, N. (2005). El aula de matemáticas como comunidad de práctica inclusiva. Educar, 32, 57-64. 
Pochulu, M., Font, V. y Rodríguez, M. (2016). Desarrollo de la competencia en análisis didáctico de formadores de futuros profesores de matemática a través del diseño de tareas. Revista Latinoamericana de Investigación en Matemática Educativa, 19(1), 71-98. https://doi.org/10.12802/relime.13.1913

Pujolàs, P. (2009). Aprendizaje Cooperativo y Educación Inclusiva: Una forma práctica de aprender juntos alumnos diferentes. En R. Blanco (Ed.), VI Jornadas de cooperación educativa con Iberoamérica sobre educación especial e inclusión educativa. Estrategias para el desarrollo de escuelas y aulas inclusivas. Santiago de Chile: Oficina Regional de Educación para América Latina y el Caribe.

Sáenz, C. y Lebrija, A. (2014). La formación continua del profesorado de matemáticas: Una práctica reflexiva para una enseñanza centrada en el aprendiz. Revista Latinoamericana de Investigación en Matemática Educativa, 17(2), 219-244. https:// doi.org/10.12802/relime.13.1724

Saravia, L. y Flores, I. (2005). La formación de maestros en América Latina. Estudio realizado en diez países. (R. Zariquiey, Ed.). Perú: PROEDUCA.

Spratt, J. y Florian, L. (2015). Inclusive pedagogy: From learning to action. Supporting each individual in the context of «everybody». Teaching and Teacher Education, 49, 89-96. https://doi.org/10.1016/j.tate.2015.03.006

Wieringa, R. (2014). Design Science Methodology for Information Systems and Software Engineering. London: Springer. https://doi.org/10.1007/978-3-662-43839-8 